\title{
METUIGA "METODOLOGÍA PARA EL DISEÑO DE SISTEMAS BASADOS EN INTERFACES TANGIBLES DE USUARIO Y TÉCNICAS DE GAMIFICACIÓN" EN LA ENSEÑANZA DE PORCIONES MATEMÁTICAS PARA NIÑOS CIEGOS
}

Luis R. Ramos-Aguiar

roberto.ramos.nay@gmail.com

Francisco J. Álvarez-Rodríguez

fjalvar@correo.uaa.mx

Centro de Ciencias Básicas,Universidad Autónoma de Aguascalientes,Aguascalientes, Aguascalientes, México.

\section{RESUMEN}

A lo largo de su vida las personas con problemas visuales tienen retos significativos durante su proceso de aprendizaje, especialmente en las etapas más tempranas de este proceso. Se han desarrollado diferentes herramientas tecnológicas en las últimas décadas para apoyar a personas con problemas visuales cuando interactúan con computadoras. Sin embargo, aún existe una gran brecha tecnológica para este tipo de usuarios, específicamente en el diseño de estos sistemas los cuales en su mayoría no explotan la principal herramienta de las personas con problemas visuales para percibir el mundo "sus manos". En esta investigación nos enfocamos en generar herramientas digitales siguiendo la metodología METUIGA utilizando interfaces tangibles de usuario y técnicas de gamificación; Se construyó una aplicación para la enseñanza de porciones matemáticas que incorpora la metodología planteada, utilizando los nuevos métodos para la implementación de interfaces tangibles de usuario y técnicas de gamificación. En esta investigación se realizaron pruebas de usabilidad obteniendo resultados satisfactorios.

\section{PALABRAS CLAVE}

Ingeniería, Interfaz Tangible, Modelo, Software, Desarrollo, Gamificación, Niños ciegos.

\section{METUIGA "METHODOLOGY FOR THE DESIGN OF SYSTEMS BASED ON TANGIBLE USER INTERFACES AND GAMMA TECHNIQUES" IN THE TEACHING OF MATHEMATICAL PORTIONS FOR BLIND CHILDREN}

\begin{abstract}
Throughout their lives, people with visual impairment present significant challenges during their learning process, especially in the early stages of this process. Different technological tools have been developed in the last decades to support visually impaired people when interacting with computers. However, there is still a big technological gap for this type of users, specifically in the design of these systems which mostly do not exploit the main tool of visually impaired people to perceive the world "their hands". In this research we focused on generating digital tools following the METUIGA methodology using tangible user interfaces and gamification techniques. An application was built for teaching mathematical portions that incorporates the proposed methodology, using the new methods for the implementation of tangible user interfaces and gamification techniques. In this research, usability tests were carried out with satisfactory results.
\end{abstract}

\section{KEYWORDS}

Blind Children, Development, Engineering, Gamification, Tangible Interface, Model, Software. 


\section{INTRODUCCIÓN}

Durante muchos años, la investigación sobre la Interacción Humano-Computadora (IHC) se ha centrado en las Interfaces Gráficas de Usuario (IGU), que permiten la interacción con objetos digitales a través de la pantalla, el teclado y el ratón de la computadora. Esto ha hecho que la mayoría de las modalidades de interacción utilizadas para los juegos educativos exploten en gran medida el canal visual para presentar y mostrar los contenidos educativos con el fin de permitir a los estudiantes aprenderlos por imitación. Desafortunadamente, este enfoque no es adecuado para niños con discapacidad visual. Por lo tanto, se deben proponer soluciones alternativas pero equivalentes más accesibles y adecuadas para aquellos que no pueden ver.

Recientemente, un nuevo campo de investigación que involucra interfaces tangibles ha evolucionado fluidamente, inspirando a los desarrolladores e investigadores de IHC a reemplazar los elementos de la interfaz gráfica con objetos intuitivos y cotidianos. Convirtiéndose en una gran herramienta para la enseñanza de temas educativos a personas con problemas visuales, debido a que a lo largo de su vida se les enseña a construir, manipular y navegar por el mundo físico utilizando el sentido del tacto a través de sus manos.

En este artículo presentamos una aplicación para la enseñanza de porciones matemáticas en niños con problemas visuales entre 6 y 10 años. Para la construcción de la aplicación se utilizó la metodología METUIGA la cual tiene como objetivo la construcción de sistemas interactivos utilizando interfaces tangibles de usuario y técnicas de gamificación.

El resto del documento está organizado de la siguiente manera: La Sección 2 presenta información relacionada con las interfaces tangibles de usuario. La sección 3 describe el concepto de gamificación. La sección 4 presenta la metodología propuesta. La sección 5 presenta los prototipos desarrollados. La sección 6 presenta los resultados obtenidos y finalmente, la sección 7 presenta las conclusiones y trabajos futuros.

\section{ANTECEDENTES}

Debido a la inexistencia de una metodología formal para el diseño de sistema interactivos utilizando interfaces tangibles y técnicas de gamificación y mucho menos relacionadas con niños con problemas visuales, donde se analicen aspectos del usuario en un contexto como la educación. Realizamos una investigación de diferentes metodologías para el diseño de sistemas interactivos con distintos enfoques (Centrados en el usuario, Juegos educativos, Juegos serios, software colaborativo, objetos de aprendizaje) con el propósito de identificar aquellas características que podrían ayudar en nuestra propuesta metodológica.

La primera metodología analizada fue MPIu+a (Saltiveri, 2004). Esta metodología está orientada hacia el diseño de sistemas interactivos centrados en el usuario. El modelo propuesto tiene las siguientes fases: análisis de requisitos, diseño, implementación, lanzamiento, prototipado y evaluación. Uno de los aspectos importantes de la propuesta es integrar la ingeniería de software con los principios de ingeniería de usabilidad y accesibilidad, proporcionando una metodología capaz de guiar a los equipos de desarrollo durante el proceso de implementación de un determinado sistema interactivo.

La segunda metodología analizada lleva por nombre LEGADEE (LEarning, GAme, DEsign Environment) (Marfisi-Schottman, 2012). Es una herramienta útil para ayudar al diseño de juegos educativos. El objetivo de la metodología es facilitar la colaboración entre los diferentes actores quiénes deben intervenir durante el diseño de un juego. La metodología está compuesta de varios bloques que corresponden a la secuencia de las fases que representan el proceso general de la creación del juego.

Nuestra tercera metodología analizada fue DODDLE (Document-Oriented Design and Development of Experimental Learning) (McMahon, 2009). Es una metodología de creación de juegos serios centrada en documentos. Las 4 etapas que involucra para la creación de juegos serios son: Análisis, propuesta de diseño, documentación de diseño y documentación de producción. Para validar el modelo, los autores propusieron a los estudiantes nuevos utilizarla para la concepción de un juego serio. Después las observaciones obtenidas, han notado que el vocabulario que provee es preciso para poder comunicarse entre los diferentes roles. Además, DOODLE ha obtenido un efecto positivo sobre la optimización del tiempo de producción, la calidad educativa y lúdica del juego. 
Nuestra cuarta metodología analizada fue MECONESIS (Metodología para el Diseño de Juegos Serios para Niños con Implante Coclear) (Cano Mazuera, 2016). Es una metodología que toma en cuenta habilidades cognitivas del participante como un aspecto que podría servir de ayuda para la adecuación de una interfaz adaptable a las necesidades del niño. Además, es una metodología que sigue la filosofía de Diseño centrado en el usuario, tomando al usuario final desde las diferentes etapas de la metodología.

Nuestra quinta metodología analizada fue MPOBA (Modelo de proceso para el desarrollo de objetos de aprendizaje) (Massa et al., 2011). En esta metodología se integraron técnicas y métodos propios del Diseño Centrado en el usuario con la naturaleza y metas educativas de estos objetos digitales. Los resultados obtenidos de la aplicación de este modelo determinaron cambios sustanciales que fueron valorados positivamente por los estudiantes. Esta metodología tiene por objetivo mejorar la calidad de los objetos de aprendizaje, enfatizando la evaluación de los atributos de usabilidad.

Por último, se analizó la metodología MICEE (Metodología para software colaborativo educativo para la inclusión de personas con discapacidad considerando la UX) (Serna et al., 2019). Es una metodología que consta de cuatro fases de desarrollo (Conocer, Formar, Prototipar, Medir). En la fase conocer se especifican necesidades del sistema por parte de los usuarios, para esto se identifica el contexto físico, ambiental, preferencial, emocional y cognitivo de cada tipo de usuario. La etapa Formar busca comprender los requisitos del sistema para identificar los que se implementaran en el sistema para esto se genera un prototipo que contendrá las características de contenido educativo, colaboración y UX. La fase Construir se desarrolla por medio de código el sistema con las características de contenido educativo, inclusión, colaboración y UX. En la fase medir se verifica y valida el sistema. Por último, la característica explorar corresponde a la etapa de mantenimiento del ciclo de vida llamado cascada.

La mayoría de las metodologías analizadas (MPlu+a, LEGADEE, DODDLE, MPOBA) muestran que han sido aplicadas en diferentes contextos de uso educativo con usuarios promedios. Lo que indica que no han involucrado desde la etapa inicial a personas con algún tipo de discapacidad a excepción de las metodologías MICEE y MECONESIS. Otro punto importante es que no contemplan el uso de interfaces tangibles apoyadas por técnicas de gamificación.

\section{METODOLOGÍA METUIGA}

METUIGA (Metodología para el diseño de sistemas basados en interfaces tangibles de usuario y técnicas de gamificación) pretende ser una metodología que siga la filosofía de diseño centrado en el usuario y su objetivo principal sea el diseño de sistemas interactivos para niños con problemas visuales.

En el diseño de sus productos se deben tomar en cuenta dos objetivos complementarios, un objetivo táctil con la finalidad de ofrecer herramientas que puedan ser manipulables para el niño y otorgar retroalimentación conforme va progresando a través de audio, por último se deberán implementar escenarios de progresión que utilicen técnicas de gamificación, con el interés de ofrecer un entorno favorable de aprendizaje, donde se tenga en cuenta diferentes aspectos, como: retos, puntuación, recompensas, reglas, entre otros.

Para entender cómo es que se obtuvo la metodología METUIGA, vamos a definir primeramente el significado de método y metodología. Según (Pérez \& Gardey, 2008) definen al método como un medio utilizado para llegar a un fin; por otro lado, (Hurtado \& Toro, 2007) define metodología como el estudio de los modos o maneras de llevar a cabo algo, es decir, el estudio de los métodos.

La metodología METUIGA es el resultado de una serie de análisis e investigaciones realizadas con el objetivo de generar una metodología enfocada en el desarrollo de sistemas interactivos para usuarios ciegos siguiendo los principios de diseño centrado en el usuario y el ciclo de vida en cascada iterativo, además contara con herramientas para utilizar técnicas de gamificación e interfaces tangibles en el proyecto.

Algunos de los aspectos importantes que se consideraron al momento de diseñar la metodología fueron:

1. Definir una herramienta para lograr la interacción tangible la cual forme parte de la metodología para facilitar a los futuros desarrolladores la búsqueda de alguna herramienta para este propósito.

2. Definir un proceso de gamificación capaz de producir sistemas que enganchen a los usuarios objetivos.

3. Definir evaluaciones que se realicen al finalizar cada etapa de la metodología para medir la usabilidad del sistema y el cumplimiento de los objetivos planteados.

4. METUIGA está organizada en etapas que determinan la etapa de desarrollo en la cual nos encontramos. Ver figura 1 


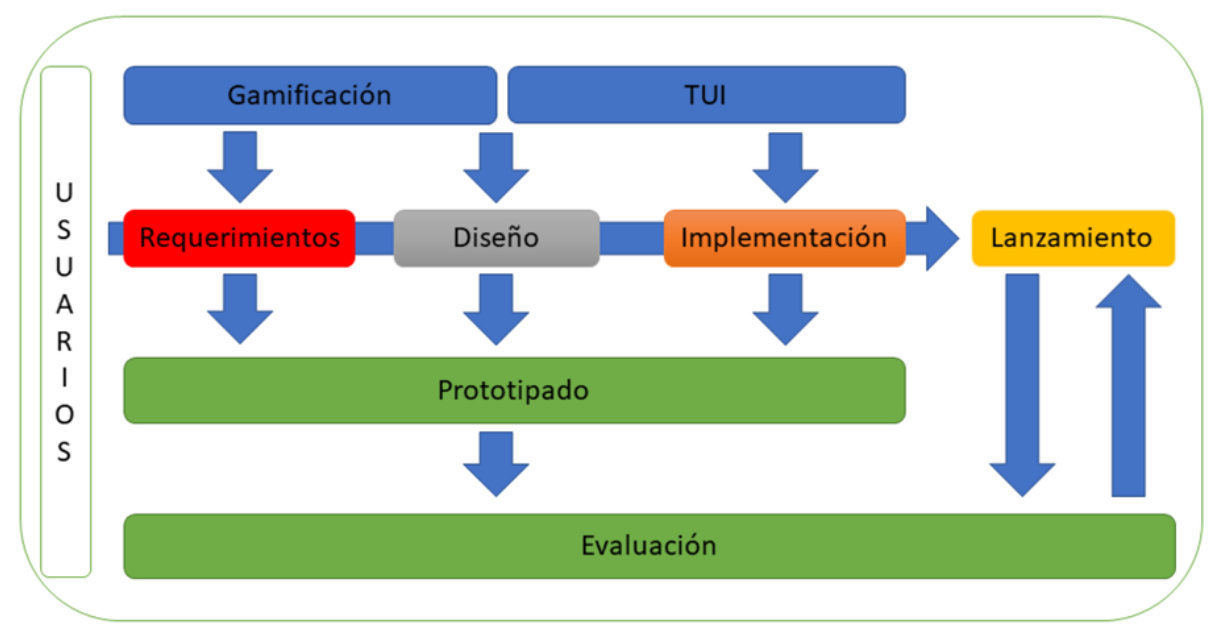

Fig. 1. Metodología para el diseño de sistemas basados en interfaces de tangibles de usuario y técnicas de gamificación para personas con problemas visuales (METUIGA). Fuente: Creación propia.

A continuación, se explica en que consiste cada etapa y las actividades que se implementan:

\section{Usuarios}

Un proceso de Diseño Centrado en el Usuario debe dejar claro de que es así sólo con mirar el esquema la primera vez. Esto es lo que queda reflejado al disponer a los usuarios en la parte izquierda abarcando el resto de las etapas de todo el proceso, en este caso, al ser el usuario alguien con problemas visuales es de gran importancia que tenga contemplado desde etapas tempranas en el proceso de desarrollo.

\section{Gamificación}

La etapa de gamificación trabaja en paralelo con las etapas de requerimientos y diseño con el objetivo de guiar a los equipos de desarrollo en la correcta selección de las mecánicas y dinámicas a implementar en base a las necesidades presentadas por el usuario en el proyecto.

\section{Interfaces tangibles de usuario}

La etapa de interfaces tangibles de usuario provee herramientas necesarias para que el equipo de desarrollo sea capaz de implementar este tipo de tecnología. Facilitando un esquema de construcción para la interfaz tangible de usuario y aconsejando herramientas de software para facilitar la detección de objetos y desarrollo del sistema interactivo.

\section{Prototipado}

Esta etapa se involucra desde la etapa inicial de metodología, dado que desde el comienzo del desarrollo de un sistema se necesita probar partes el mismo con multitud de objetivos para: Verificar funcionalidades, averiguar aspectos relacionados con la interfaz del sistema, validar la navegación, probar nuevas posibilidades de técnicas, entre otros.

\section{Evaluación}

Esta etapa se involucra desde la etapa inicial de la metodología, su objetivo consiste en probar algo. Tanto para saber si funciona correctamente o no, si cumple con las expectativas o no, o simplemente para conocer cómo funciona una determinada herramienta. La evaluación es un punto clave para la obtención de sistemas interactivos usables y accesibles. En esta etapa se aplican técnicas necesarias para recibir la retroalimentación por parte de los usuarios. También hace relación a las métricas de usabilidad y métodos de evaluación.

\section{Requerimientos}

La comunicación con los usuarios es un aspecto prioritario para las empresas que desarrollan sistemas de software, además las necesidades y las experiencias de los usuarios pueden cambiar. Durante esta etapa es importante dejar en claro el alcance y clasificación de gamificación que tendrá el sistema en base a las necesidades del usuario. 
Tabla 1. Etapa Requerimientos metodología METUIGA en modo tabla basado en las mejores prácticas (Saltiveri, 2004); (Serna et al., 2019); (Agut, 2000);(Sommerville, 1997); (Pressman, 2005);

\begin{tabular}{|l|l|}
\hline \multicolumn{1}{|c|}{ Etapa } & \multicolumn{1}{c|}{ Actividad } \\
\hline 1. Variables de trabajo & $\begin{array}{l}\text { Entrevistar a los usuarios finales de la aplicación para } \\
\text { conocer las necesidades del sistema }\end{array}$ \\
\hline 2. Requerimientos & $\begin{array}{l}\text { Identificar los requerimientos y restricciones } \\
\text { principales del sistema a desarrollar }\end{array}$ \\
\hline 3.Características & $\begin{array}{l}\text { Identificar las características de gamificación del } \\
\text { proyecto }\end{array}$ \\
\hline 4. Casos de uso y actores involucrados & $\begin{array}{l}\text { Generación del caso de uso general y actores } \\
\text { involucrados en el sistema. }\end{array}$ \\
\hline 5. Requisitos de usuario & $\begin{array}{l}\text { Identificar los requerimientos funcionales y no } \\
\text { funcionales }\end{array}$ \\
\hline
\end{tabular}

\section{Diseño}

En esta etapa se diseña la mejor solución posible, considerando que el problema fue claramente definido en la etapa de requerimientos. Sus etapas cubren diferentes funcionalidades, el diseño de la actividad, diseño de la información, y diseño de la interfaz tangible, así como las principales actividades que conforman el proceso global de los sistemas interactivos, interfaces tangibles de usuario y técnicas de gamificación.

Tabla 2. Etapa Diseño metodología METUIGA en modo tabla basado en las mejores prácticas (Zichermann \& Cunningham, 2011); (Lozano et al., 2018);(Chorney, 2012);(Serna et al., 2019);(Saltiveri, 2004);

\begin{tabular}{|l|l|}
\hline \multicolumn{1}{|c|}{ Etapa } & \multicolumn{1}{c|}{ Actividad } \\
\hline 1.Desafios y misiones & $\begin{array}{l}\text { Identificar los desafíos y misiones a implementar } \\
\text { en el sistema gamificado }\end{array}$ \\
\hline 2. Clasificación de misiones & $\begin{array}{l}\text { Clasificar las misiones comunes, individuales y } \\
\text { colectivas }\end{array}$ \\
\hline 3. Estructura de gamificación & Diseñar la Estructura de gamificación del sistema \\
\hline 4. Avatar del sistema & Diseñar la interfaz de un avatar para el sistema \\
\hline 5. Diagramas de clase & Diseñar los diagramas de clase del sistema \\
\hline 6. Diagramas de flujo & $\begin{array}{l}\text { Diseñar los diagramas dinámicos del flujo del } \\
\text { sistema }\end{array}$ \\
\hline 7. Diseños estáticos de interfaces & $\begin{array}{l}\text { Construir los diseños estáticos de las interfaces } \\
\text { involucrando a los usuarios finales y } \\
\text { considerando las mecánicas y dinámicas a } \\
\text { implementar }\end{array}$ \\
\hline 8. Diseños dinámicos de interfaces & $\begin{array}{l}\text { Construir los diseños dinámicos de las interfaces } \\
\text { involucrando a los usuarios finales }\end{array}$ \\
\hline 9. Construcción de la base de datos & $\begin{array}{l}\text { Realizar el modelado y construcción del diseño } \\
\text { de base de datos }\end{array}$ \\
\hline $\begin{array}{l}\text { 10. Diseño de seguridad de base } \\
\text { de datos }\end{array}$ & $\begin{array}{l}\text { Realizar el diseño de seguridad de la base de } \\
\text { datos }\end{array}$ \\
\hline 11. Clasificación de objetos tangibles & $\begin{array}{l}\text { Diseñar y clasificas los objetos tangibles } \\
\text { considerando la información de las interfaces } \\
\text { diseñadas }\end{array}$ \\
\hline 12. Construcción de interfaz tangible & $\begin{array}{l}\text { Diseñar y construir la interfaz tangible en base a } \\
\text { las recomendaciones de la metodología }\end{array}$ \\
\hline
\end{tabular}

\section{Implementación}

También conocida como la etapa de codificación, ya que es donde se debe escribir el código software necesario que hará posible que el sistema finalmente implementado cumpla con las especificaciones establecidas en la etapa de requerimientos y responda al diseño del sistema. 
Tabla 3. Etapa Implementación metodología METUIGA en modo tabla basada en las mejores prácticas (Serna et al., 2019); (Saltiveri, 2004); (Grau \& Segura, 2008);

\begin{tabular}{|l|l|}
\hline \multicolumn{1}{|c|}{ Etapa } & \multicolumn{1}{c|}{ Actividad } \\
\hline 1. Estándar de codificación & $\begin{array}{l}\text { Identificar un estándar de codificación para la } \\
\text { programación del sistema }\end{array}$ \\
\hline 2. Aplicación del estándar & Aplicar el estándar de codificación seleccionado \\
\hline 3. Diagrama de organización & $\begin{array}{l}\text { Diseñar un diagrama de organización de los } \\
\text { componentes y módulos en el sistema }\end{array}$ \\
\hline 4. Diagrama de estructura & $\begin{array}{l}\text { Diseñar un diagrama de estructura de directorios y } \\
\text { archivos finales del sistema }\end{array}$ \\
\hline 5. Contenido de carpetas & $\begin{array}{l}\text { Construir una tabla de Información de contenido } \\
\text { de carpetas del sistema }\end{array}$ \\
\hline
\end{tabular}

\section{Lanzamiento}

En esta etapa se comprueba que se ha conseguido la aceptabilidad del sistema, mediante una correcta combinación de aceptabilidad social y práctica. En esta etapa es importante tener una retroalimentación del usuario a través de pruebas. Una vez concluida la etapa de evaluación, el software podrá ser lanzado al usuario.

Tabla 4. Etapa Lanzamiento metodología METUIGA en modo tabla basada en las mejores prácticas (Brooke, 1996); (Pino et al., 2006); (Nielsen, 1993);

\begin{tabular}{|l|l|}
\hline \multicolumn{1}{|c|}{ Etapa } & \multicolumn{1}{c|}{ Actividad } \\
\hline 1. Evaluar usabilidad según los usuarios & $\begin{array}{l}\text { Identificar la usabilidad del sistema según los } \\
\text { usuarios }\end{array}$ \\
\hline 2. Evaluar objetivos del sistema & $\begin{array}{l}\text { Identificar la evaluación de los objetivos por } \\
\text { parte de expertos en el área }\end{array}$ \\
\hline 3. Evaluar usabilidad heurística & $\begin{array}{l}\text { Identificar la usabilidad de forma heurística del } \\
\text { sistema según los usuarios }\end{array}$ \\
\hline
\end{tabular}

\section{APLICACIÓN DE LA METODOLOGIA METUIGA}

Se ha desarrollado una aplicación que tienen como objetivo apoyar en el aprendizaje de niños ciegos en el tema de porciones matemáticas. Se eligió este tema debido a que para un alumno de Primaria con deficiencias visuales, el concepto de fracción es algo bastante complejo ya que no se puede ayudar de su representación gráfica visual (Núñez, 2017).

La aplicación está compuesta de tres componentes: un sistema de seguimiento tangible, Objetos tangibles, y una aplicación. Los detalles de estos componentes son los siguientes:

\subsection{SISTEMA DE SEGUIMIENTO TANGIBLE}

Para detectar y seguir los objetos nosotros utilizamos ReacTIVision (Reactivision, 2020) el cual es un marco de visión de computadora multiplataforma de código abierto para el seguimiento rápido y robusto de marcadores fiduciales adheridos a objetos físicos, así como para el seguimiento de dedos multitáctil

(Ver Fig. 2).

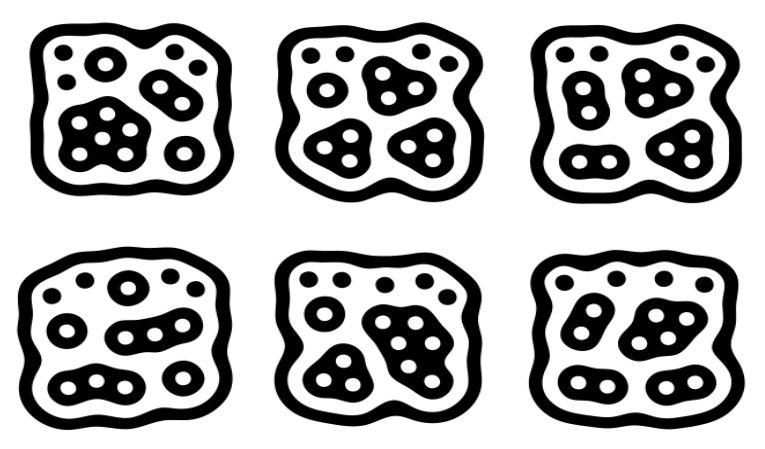

Fig. 2. Marcadores Fiducia Fuente: (Reactivision, 2020). 
Para la configuración del hardware para el sistema de seguimiento adoptamos la configuración que nos sugirió la METUIGA (Fig. 4) que consta de un acrílico de $40 \mathrm{~cm}$ x $30 \mathrm{~cm}$, una lámpara de 5v y para detectar los objetos montamos una cámara de alta definición marca Microsoft. Ver. Fig.3

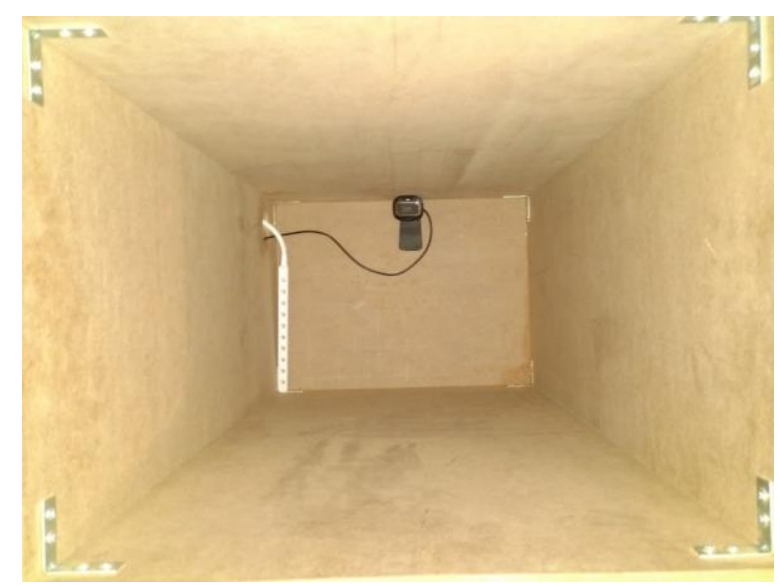

Fig. 3. Hardware de nuestro sistema tangible. Fuente: Creación propia.

\subsection{OBJETOS TANGIBLES}

Utilizamos figuras elaboradas de cartón las cuales representan diferentes porciones matemáticas (1/2, 1/3, 1/4, etc.). Estos objetos son de gran importancia debido a que, para los estudiantes ciegos, los objetos trasmiten diferentes porciones matemáticas con los cuales pueden crear imágenes mentales de las mismas. Por último, se asoció un marcador fiducia único en cada cara de cada objeto para identificarlo en nuestro sistema computacional.

\subsection{PORCIONES TANGIBLES}

La aplicación desarrollada siguiendo la metodología METUIGA lleva por nombre "Porciones tangibles" ha sido desarrollada con el objetivo de permitir al niño aprender sobre fracciones matemáticas. Las cuales están disponibles en la aplicación y son identificadas por medio de su marcador correspondiente.

La interacción con el sistema es bastante simple ya que el niño solo tiene que tomar literalmente el objeto y ponerlo sobre la mesa dependiendo de la actividad, posteriormente recibirá retroalimentación vía audio relacionada con su desempeño.

La actividad principal de la aplicación consta de una serie de preguntas sobre diferentes porciones matemáticas las cuales van aumentando de dificultad conforme va acertando, una vez que el niño identifica la respuesta y pone el objeto que él considera correcto sobre la interfaz tangible el sistema evalúa su respuesta y le comunica su resultado.

Las siguientes preguntas son las que el niño debe responder cuando está utilizando esta actividad.

1. Con que figura representas $1 / 2$ de un circulo

2. Con que figura representas $1 / 3$ de un circulo

3. Con que figura representas $1 / 4$ de un circulo

4. Con que figura representas $2 / 3$ de un circulo

5. Con que figura representas $2 / 4$ de un circulo

6. Con que figura representas $3 / 4$ de un circulo

7. Con que figura representas un entero de un circulo

8. Con que figura representas un entero y un tercio de un circulo

A continuación, se muestran los avances conseguidos hasta el momento en la construcción del software y la interfaz tangible siguiendo la metodología METUIGA. 


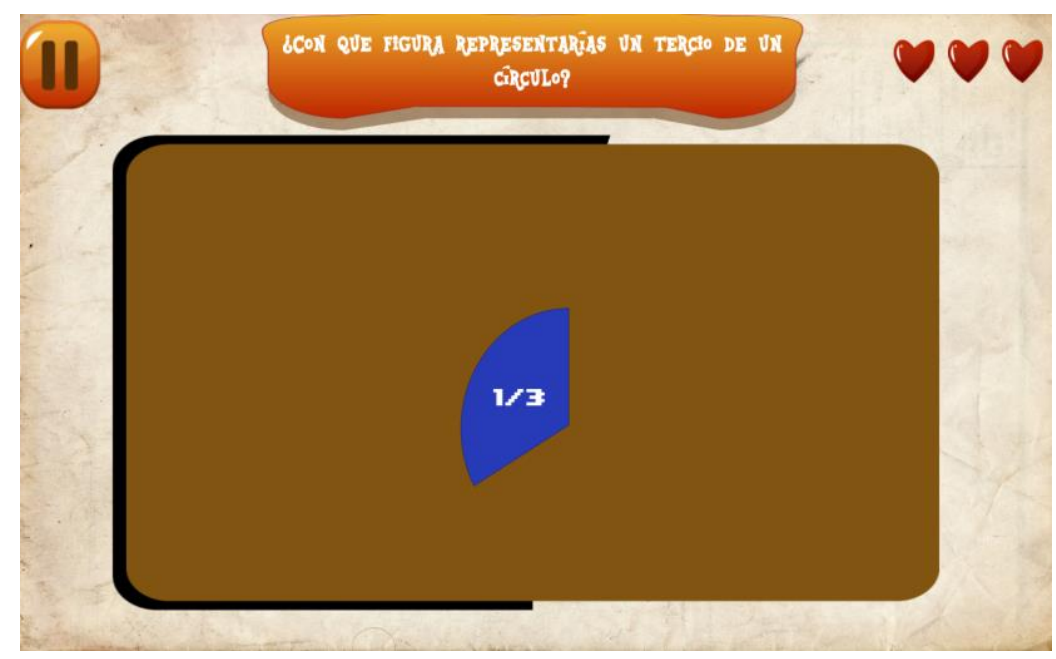

Fig. 4. Pantalla principal de la aplicación. Fuente: Creación propia.

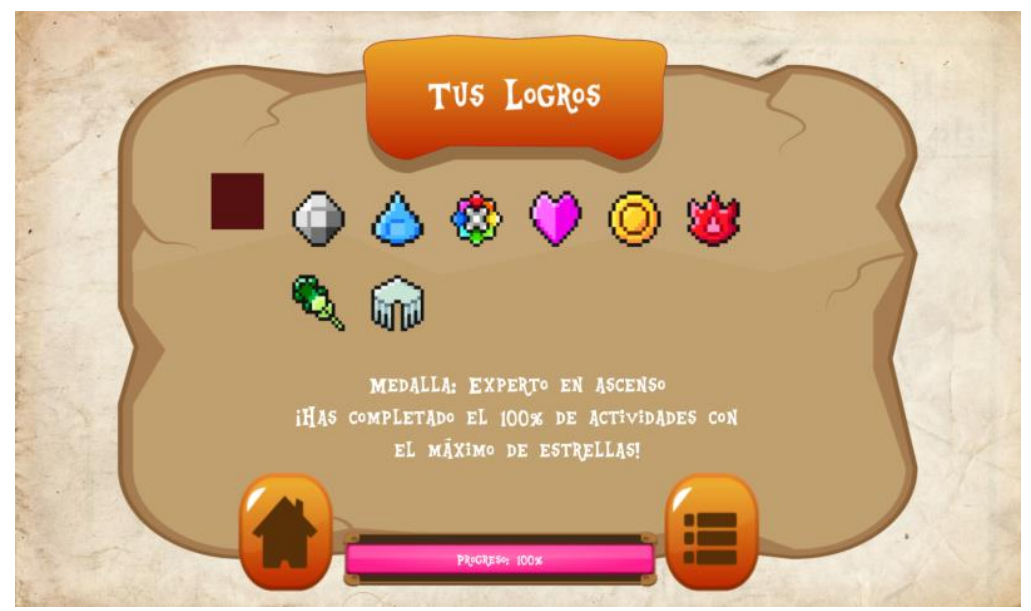

Fig. 5. Implementación de técnicas de gamificación (colección de medallas) Fuente: Creación propia.

\section{EVALUACION PRELIMINAR DEL SISTEMA}

Realizamos una prueba preliminar en la aplicación para medir la satisfacción de los usuarios al momento de utilizarlos.

\subsection{Participantes y contexto}

Para las primeras pruebas de evaluación solo se pudo reclutar a cuatro participantes normo visuales entre 6 y 10 años. La evaluación fue realizada en un entorno informal. Ellos utilizaron una la aplicación en supervisión de sus padres, posteriormente realizaron el cuestionario para conocer sus experiencias en cuanto a la satisfacción obtenida.

\subsection{Métricas de usabilidad.}

Para la medición de la satisfacción del sistema, se utilizó el instrumento System Usability Scale (SUS) (Brooke, 1996). Este cuestionario se compone de diez declaraciones relacionadas al uso del sistema. En este caso, nosotros adaptamos el cuestionario en relación con los diferentes sistemas y tipos de usuario. Los usuarios tenían que indicar en una escala de 0 a 5 el nivel de acuerdo o de desacuerdo que tenían con las declaraciones presentadas.

Según el instrumento SUS (Brooke, 1996) la obtención de resultados superiores a 68 se consideran por encima del promedio, indicando que tiene un nivel de satisfacción aceptable. En caso de que ese resultado se encuentre por debajo del promedio el sistema no cuenta con una satisfacción deseada. 
La tabla 5 muestra los resultados obtenidos utilizando la SUS por participante. El valor final está entre 0 y 100, siendo 100 el más alto grado de satisfacción en el usuario. Con una media de 95 en el SUS, se puede concluir que el nivel de satisfacción fue alto.

Tabla 5. Resultados de satisfacción por participante. Fuente: Creación propia.

\begin{tabular}{|c|c|}
\hline Participante & SUS Score \\
\hline 1 & 97.5 \\
\hline 2 & 100 \\
\hline 3 & 90 \\
\hline 4 & 92.5 \\
\hline Media & 95 \\
\hline
\end{tabular}

\section{CONCLUSIONES}

En este trabajo de investigación se mostró la metodología METUIGA, la cual todavía está en proceso de desarrollo y tiene por objetivo apoyar en la construcción de sistemas interactivos utilizando interfaces tangibles de usuario y técnicas de gamificación.

Se empleó la metodología METUIGA en la construcción de una aplicación funcional, mediante la cual se demuestra que siguiendo la metodología se logró obtener un sistema que cumplan con características tangibles y técnicas de gamificación.

Como trabajo futuro, se pretende seguir con la experimentación de la metodología propuesta creando nuevas aplicaciones orientadas a personas con problemas visuales, así como extender las funcionalidades de la aplicación presentada con el objetivo de agregar más actividades que ayuden a mejorar las habilidades de porciones matemáticas en usuarios ciegos o con baja visión para mejorar su conocimiento sobre este tema el cual les genera mucha dificultad comprender.

Después de revisar los resultados de la prueba de usabilidad, el siguiente paso es realizar la misma evaluación con usuarios con problemas visuales del estado de Aguascalientes, México para comparar sus resultados con personas normo visuales y realizar los cambios correspondientes al modelo y la aplicación aquí presentada. 


\section{REFERENCIAS}

Brooke, J. (1996). SUS-A quick and dirty usability scale. In P.W.Jordan, B. Thomas, B.A. Weerdmeester, and I.L. McClelland (Eds.), Usability Evaluation in Industry (pp. 189-194). London, UK: Taylor and Francis

Cano Mazuera, S. P. (2016). Propuesta Metodológica para el Diseño de Juegos Serios para Niños con Implante Coclear (Tesis Doctora). Universidad del Cauca, Colombia.

Chorney, A. I. (2012). Taking The Game Out Of Gamification. Dalhousie Journal of Interdisciplinary Management. https://doi.org/10.5931/djim.v8i1.242

Ferré, X., \& Segura, M. (2008). Desarrollo orientado a objetos con UML. Facultad de Informática, Universidad Politécnica de Madrid. Recuperado de https://www.uv.mx/personal/maymendez/files/2011/05/umltotal.pdf

Hurtado, I., \& Toro, J. (2007). Paradigmas y métodos de investigación en tiempos de cambio. Caracas: CEC.

Saltiveri, T. G. (2004). MPIu+a. Una metodología que integra la Ingeniería del Software, la Interacción PersonaOrdenador y la Accesibilidad en el contexto de equipos de desarrollo multidisciplinares (Tesis Doctoral). Universidad de Lleida, España.

Lozano, M. D., Penichet, V. M. R., Leporini, B., \& Fernando, A. (2018). Tangible User Interfaces to Ease the Learning Process of Visually-Impaired Children. https://doi.org/10.14236/ewic/HCI2018.87

Marfisi-Schottman, I. (2012). Méthodologie, modèles et outils pour la conception de Learning Games (Doctoral dissertation). INSA de Lyon, Français.

Massa, S., De Giusti, A., \& Pesado, P. (2011). MPOBA: un Modelo de Proceso para el desarrollo de Objetos de Aprendizaje. XVII Congreso Argentino de Ciencias de La Computación.

McMahon, M. (2009). The DODDEL model: A flexible document-oriented model for the design of serious games. In Games-Based Learning Advancements for Multi-Sensory Human Computer Interfaces: Techniques and Effective Practices. https://doi.org/10.4018/978-1-60566-360-9.ch007

Monferrer, R. (2000). Especificación de Requisitos Software según el estándar de IEEE 830. Universidad Jaume I. Departamento de Informática.

Nielsen, J. (1993). Usability Heuristics. In Usability Engineering. https://doi.org/10.1016/b978-0-08-052029-2.50008-5

Núñez, M. (2017). Matemáticas para alumnos con problemas visuales (Tesis Maestría). Universidad de la rioja, España.

Pérez, J., \& Gardey, A. (2008). Definición de método. https://definicion.de/metodo/

Pino, F. J., Garcia, F., Ruiz, F., \& Piattini, M. (2006). Adaptación de las Normas ISO/IEC 12207:2002 e ISO/IEC 15504:2003 para la Evaluación de la Madurez de Procesos Software en Países en Desarrollo. IEEE Latin America Transactions. https://doi.org/10.1109/TLA.2006.1642455

Pressman, R. (2005). Ingeniería del Software - Un enfoque práctico - Sexta Edición - Ed. México: McGrawHill. In Journal of Chemical Information and Modeling. https://doi.org/10.1017/CBO9781107415324.004

Reactivision. (2020). http://reactivision.sourceforge.net/

Serna, V. V., Rodriguez, F. J. A., Arteaga, J. M., Gallegos, J. C. P., \& Robles, T. A. (2019). MICEE Methodology. Proceedings - 2019 International Conference on Inclusive Technologies and Education, CONTIE 2019. https://doi.org/10.1109/CONTIE49246.2019.00024

Sommerville, I. (2011). Software engineering 9th Edition. ISBN-10, 137035152, 18.

Zichermann, G., \& Cunningham, C. (2011). Gamification by design: Implementing game mechanics in web and mobile apps. O'Reilly Media, Inc. 


\section{NOTAS BIOGRÁFICAS}

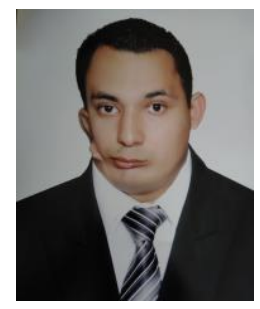

Luis Roberto Ramos Aguiar Licenciado en Sistemas computacionales por la Universidad Autónoma de Nayarit y actualmente, estudiante de Maestría en Ciencias de la Computación en la Universidad Autónoma de Aguascalientes. Su área de investigación es Ingeniería de Software.

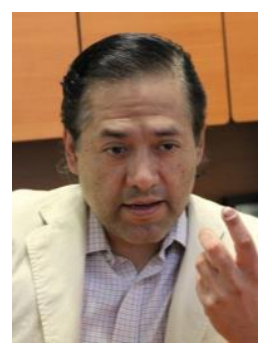

Francisco Javier Álvarez Rodríguez, Profesor de Ingeniería de Software adscrito al Departamento de Ciencias de la Computación, Universidad Autónoma de Aguascalientes (U.A.A.). Doctor en Metodología de la Enseñanza por el IMEP (México). Doctor en Ingeniería por la UNAM (México). Ha sido Decano del Centro de Ciencias Básicas en la U.A.A., así como Jefe de Departamento de Sistemas Electrónicos. Miembro de núcleos académicos de diversos posgrados de la U.A.A. Doctorado en Ciencias de la Computación, Doctorado Interinstitucional en Ciencias, Maestría en Ciencias con opción a Matemática y Computación. Autor de libros y artículos sobre la línea Objetos de Aprendizaje y Procesos de Desarrollo de Software. Actualmente es presidente del Consejo Nacional de Acreditación de programas de Informática y Computación, A.C.

\section{(ब) $\odot \odot$}

Esta obra está bajo una licencia de Creative Commons Reconocimiento-NoComercial-Compartirlgual 2.5 México. 